\title{
Convergence to No Arbitrage Equilibria in Market Games
}

Koutsougeras, Leonidas

Publication date:

2002

Document version

Early version, also known as pre-print

Citation for published version (APA):

Koutsougeras, L. (2002). Convergence to No Arbitrage Equilibria in Market Games. Department of Economics, University of Copenhagen. 


\section{DISCUSSION PAPERS Department of Economics University of Copenhagen}

$$
02-12
$$

Convergence to No Arbitrage Equilibria in Market Games

\section{Leonidas C. Koutsougeras}

Studiestræde 6, DK-1455 Copenhagen K., Denmark Tel. +45 35323082 - Fax +45 35323000 http://www.econ.ku.dk 


\title{
Convergence to No Arbitrage Equilibria in Market Games
}

\author{
Leonidas C. Koutsougeras ${ }^{\text {a,1 }}$ \\ ${ }^{a}$ School of Economic Studies, University of Manchester, Oxford Road, Manchester \\ M13 9PL, UK
}

\begin{abstract}
For market games which feature multiple posts for each commodity we show the following: (i) the 'law of one price' obtains asymptotically as the number of market participants becomes infinite, irrespectively of the characteristics of market participants; (ii) as the number of markets increases the set of equilibria is nested upwards.
\end{abstract}

JEL Classification Number: D43, D50, C72.

Key words: multiple posts, arbitrage, law of one price.

\section{Introduction}

The study of non Walrasian strategic market games of the Shapley-Shubik tradition, see (9) (3) (7), has identified two possible sources of the failure of the 'law of one price'. In (1) it was recognized that prices may be 'inconsistent', when one allows for pairwise trades among commodities, because individuals might be subject to cash constraints, which do not allow them to arbitrage

Email address: leonidas@man.ac.uk (Leonidas C. Koutsougeras).

1 Early versions of this paper were prepared while the author was a MarieCurrie Fellow at CentER, Tilburg University. The financial support of TMR no ERB4001GT965374 grant of the European Community is gratefully acknowledged. This revised version of the paper was prepared while the author was visiting the Institute of Economics, University of Copenhagen. The author is grateful to the Institute and to B. Grodal in particular, for the kind hospitality. Thanks are due to F. Germano, G. Giraud, P. Madden, J.-F. Mertens, H. Polemarchakis and to the participants of the SITE 2001 summer conference at Stanford University, for helpful comments. Responsibility for any existing errors rests with the author alone. 
prices. A different source of failure is identified in (5). In that paper the 'law of one price' fails even in the absence of liquidity constraints, because attempts to arbitrage the price difference alter market clearing prices in an adverse way, so such attempts cannot be profitable. In brief, equilibria in non Walrasian markets are compatible with arbitrage either because individuals cannot arbitrage price differences or are not interested in arbitraging the price difference. Either way, equilibria are compatible with a nonuniform distribution of equilibrium market clearing prices across markets for a commodity. This fact has been shown to be true both in the market game with numeraire (see (4)), proposed in (3), and in the market game with fiat money (see (5)) proposed in (8) and in (7).

In view of this fact, the natural question to answer is whether something can be said about the distribution of a commodity price across markets and in particular its variability across markets at any pure strategy Nash equilibrium of the market game. The main result of this paper is that, in absence of liquidity considerations, 'large' price disparities across posts for a commodity are compatible only with 'small' sets of agents. In particular, when the number of agents tends to infinity, the prices across posts for a commodity corresponding to any pure strategy Nash equilibrium, converge to a common limit regardless of the characteristics present in the economy. We believe that this is the appropriate result in this context because, in order for the correspondence 'one commodity-one price' to constitute a law, it should prevail asymptotically irrespectively of the sequence of economies and the corresponding limit. The essence of our theorem is that near the limit the law of one price does not fail drastically. Thus, the main conclusion in this paper is that the 'law of one price' is intimately related to the price taking behaviour in markets (at least by some agents), which is precisely the case in Walrasian markets.

In order to keep things in perspective, we emphasize that our result has no implication whatsoever regarding the nature of the common limit of commodity prices across different posts. In particular, this common limit need not be a competitive price. Indeed, our theorem holds true even if the limit economy is not atomless. The law of one price may be valid in the limit as long as some individuals (but not all), have negligible effects on market clearing prices. In conclusion, our result shows that an arbitrarily large number of traders implies that equilibria are characterized by uniformity of prices across posts for each commodity, but this fact by itself does not suffice to characterize perfect competition. Apparently some further qualifications are necessary for this.

It is worthwhile to draw here a distinction between our result and asymptotic convergence results to competitive equilibria, notably by (3) and by (6) (who uses the fiat money context as we do here). First of all, those results have been developed in the single trading post context, where the issue of non uniform prices does not fit. Hence, they cannot accommodate the issue of 
convergence of non uniform price distributions. An extention of those results to the multiple posts case, allows the possibility that the price in one market for a commodity converges to zero, while in another the limit is positive. For such sequences the limit is a competitive equilibrium (so a fortiori the 'law of one price' holds for the open markets), but near the limit the 'law of one price' fails drastically. Our result shows that this cannot happen (whether or not some prices are zero in the limit). In other words our result may serve to complement possible extensions of results on asymptotic convergence to competitive equilibria to the multiple posts case ${ }^{2}$ rather than being captured by them. Also notice that in the context of market games the common limit of prices could be zero, which typically is not compatible with competitive equilibria, ${ }^{3}$ yet our conclusion holds true. Finally, the same remarks apply to the results on asymptotic efficiency properties of (pure strategy) Nash equilibria shown in (8), which was also developed in the single trading post context, so it does not shed any light on the degree of non uniformity of prices at an approximately efficient equilibrium. Indeed, the (approximate) efficiency properties of equilibria with non uniform prices is an open question, which is beyond our scope here.

As it is evident in the examples in (1), the qualification for absence of liquidity constraints is necessary in the above result. Indeed, price differences may persist even in the limit if individuals cannot arbitrage price differences because of liquidity (or other institutional) constraints. In order to exemplify this possibility we sketch the construction of an equilibrium with non uniform prices, in an economy featuring a continuum of agents who face liquidity constraints (so they cannot arbitrage prices), and a finite number of agents who are not constrained (but have no interest in arbitraging prices). We can conclude then that in a frictionless context, the lack of price taking is the only source of equilibria with arbitrage.

In this paper we use the multiple posts per commodity version of the market game with fiat money. We begin our analysis, with a simple characterization of the relationship between market clearing prices across posts for a commodity. This relationship allows us to draw some interesting conclusions regarding the configuration of net trades across markets and the corresponding market clearing prices. Note that our multiple posts version of the market game allows configurations of net trades which are not possible in the standard framework. In particular, it allows individuals to buy a commodity in one market and sell it in another. We prove that precisely such a configuration of trades gives rise

$\overline{2}$ Recall that asymptotic price uniformity across posts is a necessary condition for equivalence with competitive equilibria.

3 Recall that this possibility cannot be excluded. For this reason asymptotic results had to resort to 'equilibrium points' (as in (3)), or to the statement (as in (6)) that the limit is Walrasian 'for the set of active markets', which of course can be empty. 
to non uniform market clearing prices across the markets for that commodity. This result generalizes the key idea in the example of equilibria with unequal prices across markets for a commodity, which was developed in (5). Another conceivable configuration of trades introduced by our extension of the market game, is the segregation of the economy into disjoint groups of individuals, who exchange one or more commodities among themselves in different markets. We prove that such a situation can never arise at equilibrium.

Finally, we show two results regarding the number of trading posts in a market game. Those results are rather intuitive, yet necessary for a comprehensive presentation of the market game with multiple posts per commodity. First, that the set of active equilibria of a model with any number of posts can be embedded into the set of equilibria of any model with more active trading posts. Thus, by augmenting the number of trading posts we do not 'lose' any equilibria. Second, an equilibrium for a market game with multiple posts, remains an equilibrium if we consolidate posts where prices of a commodity are equal. In particular, every equilibrium in a model with many posts where the 'law of one price' holds (namely, prices are uniform across all posts for each commodity) is an equilibrium for the single trading post model. In view of the example in (5), the inclusion of the set of equilibria of the standard game into market games with more trading posts can be strict. These last two results demonstrate that the set of equilibria with uniform prices across posts for each commodity is invariant to the structure of trading posts.

\section{The model}

Let $H$ be a finite set of agents and $L$ a set of commodity types. The consumption set of each agent is identified with $\Re_{+}^{L}$ and each individual is characterized by a preference relation, representable by a utility function $u_{h}: \Re_{+}^{L} \rightarrow \Re$, and an initial endowment $e_{h} \in \Re_{+}^{L}$. An economy is defined as $\mathcal{E}=\left\{\left(\Re_{+}^{L}, u_{h}, e_{h}\right)\right.$ : $h \in H\}$. Throughout the rest of the analysis we shall maintain the following assumptions:

Assumption $1 e_{h}>>0$ for each $h \in H$.

Assumption 2 Preferences are convex, $C^{2}$, differentiably strictly monotone ${ }^{4}$ and indifference surfaces through the endowment do not intersect the axis.

Trade in the economy is organized via a system of trading posts where individuals offer commodities for sale and place bids for purchases of commodities. A scenario for the rules of exchange is presented below.

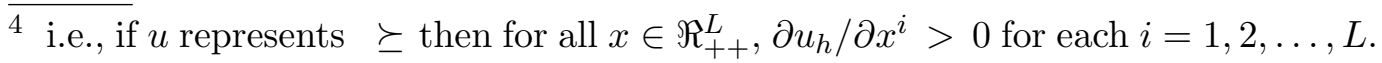




\subsection{The Market Game}

Bids for purchases of commodities are placed in terms of a unit of account. Let $K_{i}$ be a positive integer denoting the number of available trading posts for each commodity $i=1,2, \ldots, L$. The structure of the market game underlying this economy is characterized by the vector $\mathbf{k}=\left(K_{i}\right)_{i=1}^{L}$.

The strategy set of each agent consists of buy and/or sell orders in each trading post $^{5}$ :

$$
S_{h}^{\mathbf{k}}=\left\{\left(b_{h}, q_{h}\right) \in \prod_{i=1}^{L} \Re_{+}^{K_{i}} \times \prod_{i=1}^{L} \Re_{+}^{K_{i}}: \sum_{s=1}^{K_{i}} q_{h}^{i, s} \leq e_{h}^{i}, i=1,2, \ldots, L\right\} .
$$

Given a strategy profile let $B^{i, s}=\sum_{h \in H} b_{h}^{i, s}$ and $Q^{i, s}=\sum_{h \in H} q_{h}^{i, s}$. Also for each $h \in H$ define $B_{-h}^{i, s}=\sum_{n \neq h} b_{n}^{i, s}, Q_{-h}^{i, s}=\sum_{n \neq h} q_{n}^{i, s}$. Transactions in each trading post clear through the price $p^{i, s}=B^{i, s} / Q^{i, s}$. A commodity allocation is determined as follows: For each $h \in H$ and $i=1,2, \ldots, L$ :

$$
x_{h}^{i}= \begin{cases}e_{h}^{i}-\sum_{s=1}^{K_{i}} q_{h}^{i, s}+\sum_{s=1}^{K_{i}} \frac{b_{h}^{i, s}}{p^{i, s}} & \text { if } \sum_{i=1}^{L} \sum_{s=1}^{K_{i}} p^{i, s} \cdot q_{h}^{i, s} \geq \sum_{i=1}^{L} \sum_{s=1}^{K_{i}} b_{h}^{i, s} \\ e_{h}^{i}-\sum_{s=1}^{K_{i}} q_{h}^{i, s} & \text { if } \sum_{i=1}^{L} \sum_{s=1}^{K_{i}} p^{i, s} \cdot q_{h}^{i, s}<\sum_{i=1}^{L} \sum_{s=1}^{K_{i}} b_{h}^{i, s}\end{cases}
$$

where it is postulated that all divisions by zero equal zero. In other words if the value of sales covers individual bids then commodities are distributed in proportion to bids, while if an individual goes bankrupt all his/her purchases are confiscated. Consumers are viewed as solving the problem:

$$
\max _{\left(b_{h}, q_{h}\right) \in S_{h}^{\mathbf{k}}} u_{h}\left(\left(x_{h}^{i}\left(\left(b_{h}^{i, s}, q_{h}^{i, s}, B_{-h}^{i, s}, Q_{-h}^{i, s}\right)_{s=1}^{K_{i}}\right)\right)_{i=1}^{L}\right)
$$

An equilibrium is defined as a profile $\left\{\left(b_{h}, q_{h}\right) \in S_{h}^{\mathbf{k}}: h \in H\right\}$ which forms a Nash equilibrium. Notice that, due to the bankruptcy rule above, in an equilibrium with positive prices individuals can be viewed as solving the following problem:

$$
\begin{aligned}
\max _{\left(b_{h}, q_{h}\right) \in S_{h}^{\mathbf{k}}} u_{h}\left(\left(x_{h}^{i}\left(\left(b_{h}^{i, s}, q_{h}^{i, s}, B_{-h}^{i, s}, Q_{-h}^{i, s}\right)_{s=1}^{K_{i}}\right)\right)_{i=1}^{L}\right. & \\
\text { s.t. } & \sum_{i=1}^{L} \sum_{s=1}^{K_{i}} b_{h}^{i, s} \leq \sum_{i=1}^{L} \sum_{s=1}^{K_{i}} p^{i, s} \cdot q_{h}^{i, s}
\end{aligned}
$$

\footnotetext{
5 Restrictions on offer strategies could be written differently. For example one might allow individuals to 'promise' offers in each trading post. In such a case some penalty rule (e.g. confiscation of endowments) would be required to ensure feasibility at least in equilibrium.
} 
Given an economy $\mathcal{E}$ it will be useful to denote by $\mathcal{E}^{\mathbf{k}}$ the market game which is characterized by $\mathbf{k}=\left\{K_{i}\right\}_{i=1}^{L}$ trading posts. According to this notation $\mathcal{E}^{\mathbf{1}}$, where $\mathbf{1}$ is the $L$ dimensional vector with all coordinates equal to one, is the standard market game with a single trading post per commodity. Furthermore, we denote by $\mathbf{N E}\left(\mathcal{E}^{\mathbf{k}}\right)$ the set of Nash equilibrium strategy profiles of the game $\mathcal{E}^{\mathbf{k}}$ and by $\mathbf{E}\left(\mathcal{E}^{\mathbf{k}}\right)$ the set of consumption allocations which correspond to the elements of $\mathbf{N E}\left(\mathcal{E}^{\mathbf{k}}\right)$.

In what follows it will be important to understand the role of liquidity constraints in this model. Individuals who face liquidity constraints may not be able to arbitrage prices across posts for a commodity. To see this consider a situation where an individual offers all of his endowment in trading post $r$ and bids for the same commodity in another trading post $s$. Such an individual may not be able to arbitrage prices across trading posts $r$ and $s$. We now formalise this situation as follows. Given a strategy $\left(b_{h}, q_{h}\right) \in S_{h}^{\mathbf{k}}$, let $K_{i}^{b}(h)=\left\{s \in K_{i}: b_{h}^{i, s}>0\right\}$ and $K_{i}^{q}(h)=\left\{s \in K_{i}: q_{h}^{i, s}>0\right\}$. For a given strategy profile $\left\{\left(b_{h}, q_{h}\right) \in S_{h}^{\mathbf{k}}: h \in H\right\}$, define the set:

$$
C_{i}(b, q)=\left\{h \in H: \sum_{s=1}^{K_{i}} q_{h}^{i, s}=e_{h}^{i} \text { and } K_{i}^{b}(h) \cap K_{i}^{q}(h)=\emptyset\right\}
$$

The set $C_{i}(b, q)$ consists precisely of those agents who employ a strategy such as the one described above in the markets for commodity $i$. Finally, let $U_{i}(b, q)=$ $H / C_{i}(b, q)$. With this notation we can proceed now with our analysis.

\section{Equilibria without liquidity constraints}

In order to simplify the exposition, in this section we study equilibria where $U_{i}(b, q)=H$, i.e., no agent faces binding liquidity constraint. We begin with two elementary facts that will be useful in the development. In what follows we consider an equilibrium of our economy, where at least two trading posts are active, i.e., prices are positive and there is trade. Let $z_{h}^{i, s}=b_{h}^{i, s} / p^{i, s}-q_{h}^{i, s}$ denote the net trade in commodity $i$ from trading post $s$ for an individual $h \in H$.

Fact 1 Consider a budget feasible profile $(b, q)$. For each $h \in H$ if $b_{h}^{i, s} \cdot q_{h}^{i, s}>0$, there is a budget feasible $\left(\hat{b}_{h}, \hat{q}_{h}\right)$ with $\hat{b}_{h}^{i, s} \cdot \hat{q}_{h}^{i, s}=0$ such that net trades and clearing price remain unchanged. Conversely, if $b_{h}^{i, s} \cdot q_{h}^{i, s}=0$ and $b_{h}^{i, s}+q_{h}^{i, s}>0$ there is a budget feasible $\left(\hat{b}_{h}, \hat{q}_{h}\right)$ with $\hat{b}_{h}^{i, s} \cdot \hat{q}_{h}^{i, s}>0$, which results in the same net trades and clearing price. 
ProOF: See (8).

Fact 2 Consider a budget feasible profile $(b, q)$, where $b_{h}^{i, s}=q_{h}^{i, s}=0$ and $p^{i, s}>0$. Then for each $h \in H$ there is a budget feasible $\left(\hat{b}_{h}, \hat{q}_{h}\right)$ with $\hat{b}_{h}^{i, s} \cdot \hat{q}_{h}^{i, s}>0$, which results in the same net trades and clearing price.

Proof: See appendix.

We proceed with a proposition that characterizes equilibrium prices in two trading posts for a commodity:

Proposition 1 The equilibrium prices in every pair of trading posts $(s, r)$ of a commodity $i$, satisfy the following (no-arbitrage) conditions:

$$
\left(p^{i, s}\right)^{2}=\frac{B_{-h}^{i, s}}{Q_{-h}^{i, s}} \cdot \frac{Q_{-h}^{i, r}}{B_{-h}^{i, r}} \cdot\left(p^{i, r}\right)^{2} \quad \forall h \in H
$$

Proof: Notice that the statement is trivially true if one or both prices are zero, so it remains to prove it for the case where $p^{i, s} \cdot p^{i, r}>0$.

Consider any individual $h \in H$. By facts 2 and 1 respectively, this agent can be considered active on both trading posts $r$ and $s$ for commodity $i$ and in particular, active on both sides in each post. Fix one such strategy $\left(\bar{b}_{h}, \bar{q}_{h}\right)$ that is best response to $\left(B_{-h}, Q_{-h}\right)$ and denote by $\bar{B}$ and $\bar{Q}$ the corresponding aggregates. Taking the total differential of the distribution rule we obtain:

$$
d x_{h}^{i}=\sum_{s=1}^{K_{i}} \frac{B_{-h}^{i, s} \cdot \bar{Q}^{i, s}}{\left(\bar{B}^{i, s}\right)^{2}} \cdot d b_{h}^{i, s}-\sum_{s=1}^{K_{i}} \frac{B_{-h}^{i, s}}{\bar{B}^{i, s}} \cdot d q_{h}^{i, s}
$$

Also by totally differentiating the budget constraint we obtain:

$$
\sum_{s=1}^{K_{i}} \frac{\bar{B}^{i, s} \cdot Q_{-h}^{i, s}}{\left(\bar{Q}^{i, s}\right)^{2}} \cdot d q_{h}^{i, s}-\sum_{s=1}^{K_{i}} \frac{Q_{-h}^{i, s}}{\bar{Q}^{i, s}} \cdot d b_{h}^{i, s}=0
$$

Solving (4) for $d b_{h}^{i, r}$ yields:

$$
d b_{h}^{i, r}=\sum_{s=1}^{K_{i}} \frac{\bar{B}^{i, s} \cdot Q_{-h}^{i, s} \cdot \bar{Q}^{i, r}}{\left(\bar{Q}^{i, s}\right)^{2} \cdot Q_{-h}^{i, r}} \cdot d q_{h}^{i, s}-\sum_{s \neq r} \frac{Q_{-h}^{i, s} \cdot \bar{Q}^{i, r}}{\bar{Q}^{i, s} \cdot Q_{-h}^{i, r}} \cdot d b_{h}^{i, s}
$$

Substituting (5) into (3) we further obtain: 
$d x_{h}^{i}=\sum_{s \neq r}\left[\left(\frac{B_{-h}^{i, s} \bar{Q}^{i, s}}{\left(\bar{B}^{i, s}\right)^{2}}-\frac{Q_{-h}^{i, s} B_{-h}^{i, r}\left(\bar{Q}^{i, r}\right)^{2}}{\bar{Q}^{i, s} Q_{-h}^{i, r}\left(\bar{B}^{i, r}\right)^{2}}\right) d b_{h}^{i, s}+\left(\frac{\bar{B}^{i, s} Q_{-h}^{i, s}\left(\bar{Q}^{i, r}\right)^{2}}{\left(\bar{Q}^{i, s}\right)^{2} Q_{-h}^{i, r} \bar{B}^{i, r}}-\frac{B_{-h}^{i, s}}{B^{i, s}}\right) d q_{h}^{i, s}\right]$

The equation above describes the changes in the final holdings of commodity $i$, for feasible 'shifts' in the bids and offers on the $K_{i}$ trading posts. Now, in equilibrium it must be the case that $d x^{i} \leq 0$ for all $d b_{h}^{i, s}$ and $d q_{h}^{i, s}$ where $s \neq r$. Hence, it must be:

$$
\frac{B_{-h}^{i, s} \bar{Q}^{i, s}}{\left(\bar{B}^{i, s}\right)^{2}}-\frac{Q_{-h}^{i, s} B_{-h}^{i, r}\left(\bar{Q}^{i, r}\right)^{2}}{\bar{Q}^{i, s} Q_{-h}^{i, r}\left(\bar{B}^{i, r}\right)^{2}}=0, \quad \forall s \neq r
$$

which is equivalent to:

$$
\left(p^{i, s}\right)^{2}=\frac{B_{-h}^{i, s}}{Q_{-h}^{i, s}} \cdot \frac{Q_{-h}^{i, r}}{B_{-h}^{i, r}} \cdot\left(p^{i, r}\right)^{2}
$$

Furthermore, notice that the conclusion is independent of the best response chosen for individual $h$. Since the same must be true for all individuals the frst part of our claim is proved

Finally, we have the following conclusion regarding the relationship between the prices in two trading posts for a commodity.

Corollary 1 If in equilibrium $z_{h}^{i, r}=z_{h}^{i, s}=0$ for some $h \in H$ then $p^{i, s}=$ $p^{i, r}$.

Notice that the above results do not guarantee the equality between prices in two posts. In fact, we have the following result.

Proposition 2 If in equilibrium we have $z_{h}^{i, s} \cdot z_{h}^{i, r} \leq 0$, where $\left|z_{h}^{i, s}\right|+\left|z_{h}^{i, r}\right| \neq 0$ for some $h \in H$, i.e., there is one individual who makes trades of opposite signs across the two posts. Then $p^{i, r} \neq p^{i, s}$. In particular, if $z_{h}^{i, s} \leq 0$ then $p^{i, r}<p^{i, s}$.

Proof: Suppose w.l.o.g that $z_{h}^{i, s} \leq 0$ and $z_{h}^{i, r}>0$. By fact 1 it can be assumed that $b_{h}^{i, r}>0, q_{h}^{i, s} \geq 0$ and $b_{h}^{i, s}=q_{h}^{i, r}=0$. It follows that $p^{i, s} \leq \frac{B_{-h}^{i, s}}{Q_{-h}^{i, s}}$ and $p^{i, r}>\frac{B_{-h}^{i, r}}{Q_{-h}^{i, r}}$. Using these two inequalities along with Proposition 1 we obtain the following:

$$
\left(p^{i, r}\right)^{2}=\frac{B_{-h}^{i, r}}{Q_{-h}^{i, r}} \cdot \frac{Q_{-h}^{i, s}}{B_{-h}^{i, s}} \cdot\left(p^{i, s}\right)^{2} \leq \frac{1}{p^{i, s}} \cdot \frac{B_{-h}^{i, r}}{Q_{-h}^{i, r}} \cdot\left(p^{i, s}\right)^{2}<p^{i, r} \cdot p^{i, s}
$$


Thus, we conclude that $p^{i, r}<p^{i, s}$

Remark 1 In a recent paper (2), the authors use a special case of this model and conclude that equilibrium prices are always uniform across markets. It is interesting to note that their setup excludes precisely the configuration of net trades required in the above proposition.

COROLlary 2 There is no equilibrium where for some pair of agents $h, k \in H$ and some pair of posts $r, s$ for a commodity $i$, we have that $z_{h}^{i, r} \cdot z_{k}^{i, r}<0$ and $z_{h}^{i, s}=z_{k}^{i, s}=0$.

Proof: Suppose that such an equilibrium exists and let $p^{i, r}, p^{i, s}$ be the clearing prices in the two posts. Without loss of generality suppose that $z_{h}^{i, r}>0$ and $z_{k}^{i, r}<0$. An application of proposition 2 to agent $h$ implies $p^{i, r}<p^{i, s}$. A similar application to agent $k$ implies $p^{i, r}>p^{i, s}$, a contradiction

Remark 2 The last corollary excludes the possibility of equilibria where two disjoint subsets of individuals trade a commodity among themselves, in different posts.

\subsection{Asymptotic behavior of equilibrium price distributions}

In view of the fact that equilibria are compatible with a non-uniform distribution of prices across markets for a commodity, it is natural to wonder whether something can be said about the dispersion of equilibrium price distributions. In this section we link this dispersion to the number of agents and markets.

Consider an equilibrium profile $(b, q) \in \prod_{h \in H} S_{h}^{\mathbf{k}}$ with non- uniform prices. Let $T_{i}$ denote the number of distinct positive values that the price of the $i$ th commodity obtains at the chosen equilibrium. ${ }^{6}$ We may assume, by relabeling markets if necessary, that $p^{i, 1}=\min _{r=1,2, \ldots, T_{i}} p^{i, r}$.

Define: $g^{i}(b, q)=\sup \left\{\frac{p^{i, r}}{p^{i, 1}}-1: r=1,2, \ldots, T_{i}\right\}$.

LEMma $1 g^{i}(b, q) \leq \sup _{r=1,2, \ldots, T_{i}}\left(\frac{b_{h}^{i, 1}}{B_{-h}^{i, 1}}+\frac{q_{h}^{i, r}}{Q_{-h}^{i, r}}+\frac{b_{h}^{i, 1}}{B_{-h}^{i, 1}} \cdot \frac{q_{h}^{i, r}}{Q_{-h}^{i, r}}\right), \forall h \in H$.

Proof: Fix one $h \in H$. By simple manipulation of (3) we have:

\footnotetext{
${ }^{6}$ In general, $T_{i} \leq K_{i}$. However, it may be assumed that $T_{i}=K_{i}$ if one wishes so, because the same profile will be an equilibrium if markets with the same price are consolidated and markets where the price is zero are disregarded.
} 


$$
\begin{aligned}
\frac{p^{i, r}}{p^{i, 1}} & =\frac{Q^{i, r}}{B^{i, r}} \cdot \frac{B_{-h}^{i, r}}{Q_{-h}^{i, r}} \cdot \frac{Q_{-h}^{i, 1}}{B_{-h}^{i, 1}} \cdot \frac{B^{i, 1}}{Q^{i, 1}} \\
& =\frac{Q_{-h}^{i, 1}}{Q^{i, 1}} \cdot \frac{B_{-h}^{i, r}}{B^{i, r}} \cdot\left(1+\frac{b_{h}^{i, 1}}{B_{-h}^{i, 1}}+\frac{q_{h}^{i, r}}{Q_{-h}^{i, r}}+\frac{b_{h}^{i, 1}}{B_{-h}^{i, 1}} \cdot \frac{q_{h}^{i, r}}{Q_{-h}^{i, r}}\right) \\
& \leq 1+\frac{b_{h}^{i, 1}}{B_{-h}^{i, 1}}+\frac{q_{h}^{i, r}}{Q_{-h}^{i, r}}+\frac{b_{h}^{i, 1}}{B_{-h}^{i, 1}} \cdot \frac{q_{h}^{i, r}}{Q_{-h}^{i, r}}
\end{aligned}
$$

Since the same inequality is true for each $h$, we conclude that

$\forall h \in H, \sup _{r=1,2, \ldots, T_{i}}\left(\frac{p^{i, r}}{p^{i, 1}}-1\right) \leq \sup _{r=1,2, \ldots, T_{i}}\left(\frac{b_{h}^{i, 1}}{B_{-h}^{i, 1}}+\frac{q_{h}^{i, r}}{Q_{-h}^{i, r}}+\frac{b_{h}^{i, 1}}{B_{-h}^{i, 1}} \cdot \frac{q_{h}^{i, r}}{Q_{-h}^{i, r}}\right)$

Lemma 2 Let $(b, q) \in \prod_{h \in H} S_{h}^{\mathbf{k}}$ be an equilibrium profile with a non uniform distribution of prices, i.e., $T_{i} \geq 2$ for some $i=1,2, \ldots, L$. Then:

(i) Given $\epsilon>0$, we have: $\# H \geq\left(T_{i}+1\right)\left(\frac{\sqrt{1+\epsilon}}{\sqrt{1+\epsilon}-1}\right) \Rightarrow g^{i}(b, q) \leq \epsilon$

(ii) If $\# H>T_{i}+1$ then $g^{i}(b, q) \leq \frac{\left(T_{i}+1\right)\left(2 \cdot \# H-T_{i}-1\right)}{\left(\# H-T_{i}-1\right)^{2}}$

Proof: We show the contrapositive of (i). Suppose that $g^{i}(b, q)>\epsilon$. Define $\theta_{h}=\max \left\{\frac{b_{h}^{i, 1}}{B_{-h}^{i, r}},\left(\frac{q_{h}^{i, r}}{Q_{-h}^{i, r}}\right)_{r=1}^{T_{i}}\right\}$. By lemma $1 g^{i}(b, q) \leq 2 \cdot \theta_{h}+\theta_{h}^{2}$ for all $h \in H$. It follows that $\theta_{h}>-1+\sqrt{1+\epsilon}=\eta(\epsilon), \forall h \in H$. Hence, for each $h \in H$ either $\frac{b_{h}^{i, 1}}{B_{-h}^{i, 1}}>\eta(\epsilon)$ or $\frac{q_{h}^{i, r}}{Q_{-h}^{i, r}}>\eta(\epsilon)$ for some $r=1,2, \ldots, T_{i}$. Therefore,

$\forall h \in H$, either $\frac{b_{h}^{i, 1}}{B^{i, 1}}>\frac{\eta(\epsilon)}{1+\eta(\epsilon)}$ or $\frac{q_{h}^{i, r}}{Q^{i, r}}>\frac{\eta(\epsilon)}{1+\eta(\epsilon)}$, for some $r=1,2, \ldots, T_{i}$.

Define $V=\left\{h \in H: \frac{b_{h}^{i, 1}}{B^{i, 1}}>\frac{\eta(\epsilon)}{1+\eta(\epsilon)}\right\}$ and $V_{r}=\left\{h \in H: \frac{q_{h}^{i, r}}{Q^{i, r}}>\frac{\eta(\epsilon)}{1+\eta(\epsilon)}\right\}$.

We have: $\# V \frac{\eta(\epsilon)}{1+\eta(\epsilon)}<\sum_{h \in V} \frac{b_{h}^{i, 1}}{B^{i, 1}} \leq 1$, so $\# V<\frac{1+\eta(\epsilon)}{\eta(\epsilon)}$. Similarly $\# V_{r}<\frac{1+\eta(\epsilon)}{\eta(\epsilon)}$.

Recall now that $H=\left(\bigcup_{r=1}^{T_{i}} V_{r}\right) \cup V$, so $\# H \leq \sum_{r=1}^{T_{i}} \# V_{r}+\# V<\left(T_{i}+1\right)\left(\frac{1+\eta(\epsilon)}{\eta(\epsilon)}\right)$.

Thus, we have shown that: $g^{i}(b, q)>\epsilon \Rightarrow \# H<\left(T_{i}+1\right)\left(\frac{1+\eta(\epsilon)}{\eta(\epsilon)}\right)$.

Finally, the second claim of the lemma follows directly by solving the last inequality for $\epsilon$, taking into account the hypothesis $\# H>T_{i}+1$. In this way 
we have for any $\epsilon>0$ :

$$
g^{i}(b, q)>\epsilon \Rightarrow \frac{\left(T_{i}+1\right)\left(2 \cdot \# H-T_{i}-1\right)}{\left(\# H-T_{i}-1\right)^{2}}>\epsilon,
$$

which implies the statement of claim (ii)

The implication of the last lemma for the asymptotic behavior of price disparities is crystallized in the following theorem ${ }^{7}$. Consider a sequence of economies with their associated market structures $\mathbf{k}_{n}$ where $\# H_{n} \rightarrow \infty$ and a sequence of corresponding equilibria $\left(b_{n}, q_{n}\right) \in \prod_{h \in H} S_{h}^{\mathbf{k}_{n}}$. Define $z_{n}^{i}=\# H_{n} / T_{i}^{n}+1$.

Theorem $1 z_{n}^{i} \rightarrow \infty \Rightarrow g^{i}\left(b_{n}, q_{n}\right) \rightarrow 0$.

Proof: Since $z_{n}^{i} \rightarrow \infty$ we have that eventually $z_{n}^{i}>1$, so according to the above theorem

$$
g^{i}\left(b_{n}, q_{n}\right) \leq \frac{2 z_{n}^{i}-1}{\left(z_{n}^{i}-1\right)^{2}} .
$$

Thus, as $z_{n}^{i} \rightarrow \infty$ we have $g^{i}\left(b_{n}, q_{n}\right) \rightarrow 0$

\section{Equilibria with binding liquidity constraints}

So far we have focused on equilibria where individuals were not facing liquidity constraints. In this section we qualify our resuts for equilibria where some agents may be facing binding liquidity constraints, i.e., $C_{i}(b, q) \neq \emptyset$. Intuitively, such agents may not be able to arbitrage prices because of cash constraints. First, notice that facts (1) and (2) do not apply in this case precisely because the strategies constructed in their proofs violate the liquidity constraint which requires that $\sum_{s=1}^{K_{i}} q_{h}^{i, s}=e_{h}^{i}$. In this case proposition (1) can be restated as follows:

Proposition 3 The equilibrium prices in every pair of trading posts $(s, r)$ of a commodity $i$, satisfy the following (no-arbitrage) conditions:

$$
\left(p^{i, s}\right)^{2} \leq \frac{B_{-h}^{i, s}}{Q_{-h}^{i, s}} \cdot \frac{Q_{-h}^{i, r}}{B_{-h}^{i, r}} \cdot\left(p^{i, r}\right)^{2} \quad \forall h \in C_{i}(b, q)
$$

where the inequality is strict iff $s \in K_{i}^{b}(h)$ and $r \in K_{i}^{q}(h)$.

Proof: Since $\sum_{s=1}^{K_{i}} q_{h}^{i, s}=e_{h}^{i}$, from our assumptions on preferences it follows that $K_{i}^{b}(h) \neq \emptyset$. Without loss of generality let $s \in K_{i}^{b}(h)$. Consider now the differentials of the budget constraint and the allocation rule as in proposition

$\overline{7}$ The statement of this theorem has been suggested to me by J.-F. Mertens. 
(1). In this case equation (??) has to hold for all $d b$ if $r \in K_{i}^{b}(h)$, all $d q \leq 0$ if $r \in K_{i}^{q}(h)$ and all $d b \geq 0$ if $r \notin K_{i}^{b}(h)$. Hence, the conclusion follows

Note that as a direct corollary of (3), we can ammend proposition (2) as follows:

Proposition 4 Let $(b, q) \in \mathbf{N E}\left(\mathcal{E}^{\mathbf{k}}\right)$ be an equilibrium where $C_{i}(b, q) \neq \emptyset$. If for some $h \in C_{i}(b, q)$ we have $s \in K_{i}^{q}(h)$ and $r \in K_{i}^{b}(h)$, then $p^{i, r}<p^{i, s}$.

Finally, in view of proposition (3), lemmas (1) and (2) have to be restated as follows:

Lemma $3 g^{i}(b, q) \leq \sup _{r=1,2, \ldots, T_{i}}\left(\frac{b_{h}^{i, 1}}{B_{-h}^{i, 1}}+\frac{q_{h}^{i, r}}{Q_{-h}^{i, r}}+\frac{b_{h}^{i, 1}}{B_{-h}^{i, 1}} \cdot \frac{q_{h}^{i, r}}{Q_{-h}^{i, r}}\right), \forall h \in U_{i}(b, q)$.

LEMMA 4 Let $(b, q) \in \prod_{h \in H} S_{h}^{\mathbf{k}}$ be an equilibrium profile with a non uniform distribution of prices, i.e., $T_{i} \geq 2$ for some $i=1,2, \ldots, L$. Then:

(i) Given $\epsilon>0$, \#U $(b, q) \geq\left(T_{i}+1\right)\left(\frac{\sqrt{1+\epsilon}}{\sqrt{1+\epsilon}-1}\right) \Rightarrow g^{i}(b, q) \leq \epsilon$

(ii) If $\# U_{i}(b, q)>T_{i}+1$ then $g^{i}(b, q) \leq \frac{\left(T_{i}+1\right)\left(2 \cdot \# U_{i}(b, q)-T_{i}-1\right)}{\left(\# U_{i}(b, q)-T_{i}-1\right)^{2}}$

The proofs of the above results are identical to their counterparts of the previous section with $U_{i}(b, q)$ replacing $H$.

We now sketch an example, which shows that when the number of individuals who can arbitrage prices (i.e, do not face cash constraints) remains bounded, non uniform prices may persist even in the limit. This example is reminiscent to the ones in $(1)$.

\subsection{An Example}

Let $(A, \mu)$ be a measure space, where $\mu$ is non atomic and $\mu(A)=1$. Consider an economy where $H=A \cup\{h, k\}$, i.e., the economy consists of an atomless set of agents along with two atoms. Suppose that for a given commodity the endowments of individuals are as follows: $e_{h}=7, e_{k}=5$ and $e_{t}=\frac{1}{2}, \mu$ ae $t \in A$. Individuals may exchange this commodity simultaneously in two markets labelled $r$ and $s$. Let us consider the following profile of bids and offers, where $\lambda=\frac{8}{63}$ : 


$$
\begin{aligned}
& \left(b_{h}^{r}, b_{h}^{s}, q_{h}^{r}, q_{h}^{s}\right)=\left(2,12 \lambda^{2}, \frac{7}{2}, 1\right) \\
& \left(b_{k}^{r}, b_{k}^{s}, q_{k}^{r}, q_{k}^{s}\right)=\left(2,66 \lambda^{2}, \frac{1}{2}, 1\right) \\
& \left(b_{t}^{r}, b_{t}^{s}, q_{t}^{r}, q_{t}^{s}\right)=\left(0,6 \lambda^{2}, \frac{1}{2}, 0\right) \quad t a e
\end{aligned}
$$

With this profile of strategies the market clearing prices are unequal: $p^{s} / p^{r}=$ $\frac{48}{63}<1$. Furthermore, the prevailing prices in the two markets satisfy the no arbitrage condition of proposition (1) for agents $h$ and $k$. Thus, those agents cannot arbitrage prices because, as in (5), their attempts to do so have adverse price effects. Notice that agents $t \in A$ face a liquidity constraint: they offer all their endowment for sale in trading post $r$ and bid for the commodity in the post $s$. The above profile of strategies also satisfy the no arbitrage conditions of proposition (3) for $t \in A$. These agents do not have any effect on prices in the two posts. However, neither those agents can arbitrage the price difference because, as in (1), they face a cash constraint: those agents would be interested in moving sales from the cheaper trading post $s$ to the more expensive trading post $r$ but they cannot as they already offer all their endowment in trading post $r$. Similarly, they cannot transfer bids from the expensive post $(r)$ to the cheaper post $(s)$ as they already bid zero in $r$.

From here on proceeding as in (5), we can construct strategy profiles for other commodities and find utility functions for which the above profile would be part of a Nash equilibrium one. This example shows how theorem (4) can fail when the number of individuals who do not face cash constraint (and therefore can take advantage of arbitrage opportunities) remains bounded.

\section{$5 \quad$ Equilibria with uniform prices}

Not all equilibria of the multiple trading posts version of the market game exhibit non uniform prices. Equilibria with uniform prices do exist and they are intimately related to the standard single post market game. Clearly, one can replace an inactive post with more inactive posts and reproduce the same outcome. However, it can be shown that every equilibrium allocation of the standard model with a single active post per commodity can be obtained as a uniform equilibrium of the model with multiple active trading posts ${ }^{8}$. In other words we do not 'miss' any equilibria by augmenting the number of trading posts. We provide here a proof for a game with two trading posts per commodity. The proof can be extended to the general case via an inductive argument.

\footnotetext{
$\overline{8}$ In view of the existence proof in (7) this fact is also an indirect existence proof for our model.
} 
Consider the market games $\mathcal{E}^{\mathbf{1}}$ and $\mathcal{E}^{\mathbf{2}}$ (where $\mathbf{2}$ is the $L$ dimensional vector with all coordinates equal to 2). Let $x \in \mathbf{E}\left(\mathcal{E}^{\mathbf{1}}\right)$ be an equilibrium allocation corresponding to the strategy profile $\left\{\left(b_{h}, q_{h}\right) \in S_{h}^{\mathbf{1}}: h \in H\right\}$. For each index $i=1,2, \ldots, L$ choose $0<t_{i}<1$ and consider the profile $\left\{\left(\hat{b}_{h}, \hat{q}_{h}\right) \in S_{h}^{2}: h \in\right.$ $H\}$ for the game $\mathcal{E}^{\mathbf{2}}$, where for each $h \in H$ :

$$
\left(\hat{b}_{h}^{i, s}, \hat{q}_{h}^{i, s}\right)= \begin{cases}t_{i} \cdot\left(b_{h}^{i}, q_{h}^{i}\right) & s=1 \\ \left(1-t_{i}\right) \cdot\left(b_{h}^{i}, q_{h}^{i}\right) & s=2\end{cases}
$$

Notice that this strategy profile results in a uniform distribution of prices across posts for each commodity and the same commodity allocation $x$ as in the game $\mathcal{E}^{\mathbf{1}}$.

Claim 3 The profile of strategies $\left(\hat{b}_{h}, \hat{q}_{h}\right)_{h \in H}$ constructed above is an equilibrium for the game $\mathcal{E}^{\mathbf{2}}$. In particular $x \in \mathbf{E}\left(\mathcal{E}^{\mathbf{2}}\right)$.

Proof: See appendix.

Proceeding in the same way as above one can establish the following result:

Proposition 5 Given two vectors with positive integer coordinates $\mathbf{k}, \mathbf{t} \in \Re^{L}$ where $\mathbf{t} \geq \mathbf{k}$, we have $\mathbf{E}\left(\mathcal{E}^{\mathbf{k}}\right) \subseteq \mathbf{E}\left(\mathcal{E}^{\mathbf{t}}\right)$. In particular, any equilibrium with $K_{i}$ active posts for a commodity $i$, can be obtained as an equilibrium with $T_{i}$ active posts for that commodity.

The next result establishes that the converse inclusion holds for the subset of uniform equilibria.

Proposition 6 Let $x \in \mathbf{E}\left(\mathcal{E}^{\mathbf{t}}\right)$ be uniform,i.e., there is a unique price that clears all the trading posts where each commodity is traded. Then $x \in \mathbf{E}\left(\mathcal{E}^{\mathbf{k}}\right)$ for all $\mathbf{k} \leq \mathbf{t}$. In particular, $x \in \mathbf{E}\left(\mathcal{E}^{\mathbf{1}}\right)$.

Proof: See appendix.

\section{Conclusion}

The central message of this paper is that, the 'law of one price' and its implications for the relevance of the market structure, is intimately related to the price taking hypothesis. Perhaps it would be useful to spell out, some interpretation of our results.

Propositions (2) and (4) suggest that the arbitrage activities of non price tak- 
ing individuals (selling a commodity in one market and buying it back from another) never exhausts arbitrage possibilities. Therefore, the persistence of arbitrage in markets where some individuals undertake arbitrage activities, could be used as an indicator of market power of those individuals, because arbitrage possibilities should be exhausted if those individuals were price takers.

With regards to our asymptotic results (1) and (4), note that the rate of convergence does not depend at all on individual characteristics. This reflects the intuition that 'small' agents can take advantage of an arbitrage opportunity just as well as 'large' agents. Thus, the distribution of characteristics should be irrelevant for such a result. Indeed, our result is valid even if the limit economy is not atomless. In other words our result holds true whether or not equilibria are competitive in the limit. Recall that for the 'law of one price' to hold it suffices that some individuals have negligible effects on market clearing prices, i.e., act as price takers. Of course, this is the case in a competitive market where all individuals are negligible in the formation of market clearing prices. On the other hand notice that not all agents need to be negligible in order for the 'law of one price' to hold. Therefore, the validity of the law of one price is a more general issue than the prevalence of perfect competition. The independence of our theorem from individual characteristics reflects precisely this fact.

The essence of our result is that, as we approach the limit (competitive or not), prices in different markets approach one another in an orderly fashion, so near the limit the 'law of the one price' does not fail dramatically. This is very important for the tradition of viewing the economy as a large but finite one as opposed to an idealized continuum.

Our results on equilibria with uniform prices verify that when the 'law of one price' prevails, indeed the market structure is irrelevant. However, the coexistence of equilibria with uniform and non uniform prices introduces a coordination issue: given a market game which of the two types of equilibria is likely that individuals would coordinate on? This question cannot be answered within the static Nash equilibrium model. This could be the topic of further research using more sophisticated equilibrium refinements. 


\section{APPENDIX}

Proof of Fact 2 This is a simple consequence of the fact that every individual can be viewed as making a 'wash' sale in the trading posts where he is nonactive. Suppose that for some $h \in H$ we have $b_{h}^{i, s}=q_{h}^{i, s}=0$. Given $p^{i, s}=$ $\frac{B_{-h}^{i, s}}{Q_{-h}^{i, s}}>0$, this agent can be viewed as using a strategy $\hat{b}_{h}^{i, s} \cdot \hat{q}_{h}^{i, s}>0$ such that $\frac{\hat{b}_{h}^{i, s}}{\hat{q}_{h}^{i, s}}=\frac{B_{-h}^{i, s}}{Q_{-h}^{i, s}}$. Notice that in this way $z_{h}^{i, s}=0$ and $p^{i, s}=\frac{B_{-h}^{i, s}}{Q_{-h}^{i, s}}=\frac{B_{-h}^{i, s}+\hat{b}_{h}^{i, s}}{Q_{-h}^{i, s}+\hat{q}_{h}^{i, s}}$. Thus, the allocation and price remain unchanged with this transformation in the strategy of individual $h$. It is easy to verify that the new strategy is also budget feasible

Proof of Claim 3 Suppose the claim is not true. Then for some agent $h \in H$ there exists a strategy $\left(\beta_{h}, \theta_{h}\right) \in S_{h}$ so that:

$$
u_{h}\left(\left(x_{h}^{i}\left(\beta_{h}^{i, s}, \theta_{h}^{i, s}, \hat{B}_{-h}^{i, s}, \hat{Q}_{-h}^{i, s}\right)_{s=1}^{2}\right)_{i=1}^{L}\right)>u_{h}\left(\left(x_{h}\left(\hat{b}_{h}^{i, s}, \hat{q}_{h}^{i, s}, \hat{B}_{-h}^{i, s}, \hat{Q}_{-h}^{i, s}\right)_{s=1}^{2}\right)_{i=1}^{L}\right)
$$

Without loss of generality (see fact 1) we may assume that for each commodity $i=1,2, \ldots, L, \beta_{h}^{i, 1} \cdot \theta_{h}^{i, 1}=0$ and $\beta_{h}^{i, 2} \cdot \theta_{h}^{i, 2}=0$. Note that, given our assumptions on preferences and endowments, the bankruptcy rule ensures that:

$$
\sum_{i=1}^{L} \sum_{s=1}^{2} \frac{\hat{B}_{-h}^{i, s}}{\hat{Q}_{-h}^{i, s}+\theta_{h}^{i, s}} \cdot \theta_{h}^{i, s} \geq \sum_{i=1}^{L} \sum_{s=1}^{2} \beta_{h}^{i, s}
$$

Given $\left(\beta_{h}, \theta_{h}\right)$, define: $L_{1}=\left\{i: \beta_{h}^{i, 1}>0, \beta_{h}^{i, 2}>0\right\}, L_{2}=\left\{i: \theta_{h}^{i, 1}>0, \theta_{h}^{i, 2}>0\right\}$, and $L_{3}=\left\{i: \beta_{h}^{i, s}>0, \theta_{h}^{i, r}>0, s \neq r\right\}$.

- Step I Consider the commodities $i \in L_{3}$ (i.e., the commodities where the consumer is a net buyer in one trading post and a net seller in the other).

Define a new strategy $\left(\hat{\beta}_{h}, \hat{\theta}_{h}\right) \in S_{h}$ where for $i \notin L_{3}$ we have $\hat{\beta}_{h}^{i}=\beta_{h}^{i}$, $\hat{\theta}_{h}^{i}=\theta_{h}^{i}$ while, for $i \in L_{3}, \hat{\beta}_{h}^{i, s}=t_{i} \beta_{h}^{i, s}, \hat{\beta}_{h}^{i, r}=\left(1-t_{i}\right) \beta_{h}^{i, s}$ and $\hat{\theta}_{h}^{i, s}=t_{i} \theta_{h}^{i, r}$, $\hat{\theta}_{h}^{i, r}=\left(1-t_{i}\right) \theta_{h}^{i, r}$. With this strategy the prices in the two posts of the $i$ th 
commodity would become equal:

$p^{i, s}=\frac{\hat{B}_{-h}^{i, s}+t_{i} \cdot \beta_{h}^{i, s}}{\hat{Q}_{-h}^{i, s}+t_{i} \cdot \theta_{h}^{i, r}}=\frac{t_{i} \cdot B_{-h}^{i}+t_{i} \cdot \beta_{h}^{i, s}}{t_{i} \cdot Q_{-h}^{i}+t_{i} \cdot \theta_{h}^{i, r}}=\frac{\left(1-t_{i}\right) \cdot B_{-h}^{i}+\left(1-t_{i}\right) \cdot \beta_{h}^{i, s}}{\left(1-t_{i}\right) \cdot Q_{-h}^{i}+\left(1-t_{i}\right) \cdot \theta_{h}^{i, r}}=p^{i, r}$

With this strategy the net trade of consumer $h$ is: $\beta_{h}^{i, s} \cdot\left(\frac{Q_{-h}^{i, s}+\theta_{h}^{i, r}}{B_{-h}^{i, s}+\beta_{h}^{i, s}}\right)-\theta_{h}^{i, r}$.

Notice that this net trade is at least as big as the net trade resulting from the initial strategy, i.e.,

$$
\beta_{h}^{i, s} \cdot\left(\frac{Q_{-h}^{i, s}+\theta_{h}^{i, r}}{B_{-h}^{i, s}+\beta_{h}^{i, s}}\right)-\theta_{h}^{i, r} \geq \beta_{h}^{i, s} \cdot\left(\frac{t_{i} \cdot Q_{-h}^{i, s}}{t_{i} \cdot B_{-h}^{i, s}+\beta_{h}^{i, s}}\right)-\theta_{h}^{i, r}
$$

Therefore, by shifting a proportion of the bid (offer) from the first trading post to the second and at the same time shifting the same proportion of the offer (bid) from the second to the first, the consumer can achieve an allocation which is at least as good as the original (keeping the strategy fixed in the other commodities). Notice that doing so is budget feasible because:

$$
\theta_{h}^{i, r} \cdot\left(\frac{B_{-h}^{i, s}+\beta_{h}^{i, s}}{Q_{-h}^{i, s}+\theta_{h}^{i, r}}\right) \geq \theta_{h}^{i, r} \cdot\left(\frac{\left(1-t_{i}\right) \cdot B_{-h}^{i, s}}{\left(1-t_{i}\right) \cdot Q_{-h}^{i, s}+\theta_{h}^{i, r}}\right)
$$

We conclude then that if the consumer can improve over the first allocation by a selling-buying strategy, then he can do so by either selling or buying in both trading posts. Hence, we can assume without loss of generality that $L_{3}=\emptyset$.

- Step II Consider now $i \in L_{1}$ (i.e., the commodities where the consumer is a net purchaser in both trading posts).

In this case the total net trade of commodity $i$ is given by:

$$
\beta_{h}^{i, 1} \cdot \frac{\hat{Q}_{-h}^{i, 1}}{\hat{B}_{-h}^{i, 1}+\beta_{h}^{i, 1}}+\beta_{h}^{i, 2} \cdot \frac{\hat{Q}_{-h}^{i, 2}}{\hat{B}_{-h}^{i, 2}+\beta_{h}^{i, 2}}=\beta_{h}^{i, 1} \cdot \frac{t_{i} \cdot Q_{-h}^{i}}{t_{i} \cdot B_{-h}^{i}+\beta_{h}^{i, 1}}+\beta_{h}^{i, 2} \cdot \frac{\left(1-t_{i}\right) \cdot Q_{-h}^{i}}{\left(1-t_{i}\right) \cdot B_{-h}^{i}+\beta_{h}^{i, 2}}
$$

In the game $\mathcal{E}^{\mathbf{1}}$ the same net trade could be achieved via a bid $\hat{\beta}_{h}^{i}$ that solves the equation:

$$
\hat{\beta}_{h}^{i} \cdot \frac{Q_{-h}^{i}}{B_{-h}^{i}+\hat{\beta}_{h}^{i}}=\beta_{h}^{i, 1} \cdot \frac{t_{i} \cdot Q_{-h}^{i}}{t_{i} \cdot B_{-h}^{i}+\beta_{h}^{i, 1}}+\beta_{h}^{i, 2} \cdot \frac{\left(1-t_{i}\right) \cdot Q_{-h}^{i}}{\left(1-t_{i}\right) \cdot B_{-h}^{i}+\beta_{h}^{i, 2}}
$$


which yields:

$$
\hat{\beta}_{h}^{i}=\frac{t_{i} \cdot\left(1-t_{i}\right) \cdot B_{-h}^{i} \cdot\left(\beta_{h}^{i, 1}+\beta_{h}^{i, 2}\right)+\beta_{h}^{i, 1} \cdot \beta_{h}^{i, 2}}{t_{i} \cdot\left(1-t_{i}\right) \cdot B_{-h}^{i}+\left(1-t_{i}\right)^{2} \cdot \beta_{h}^{i, 1}+t_{i}^{2} \cdot \beta_{h}^{i, 2}}
$$

In other words the agent $h$ could receive the same net trade of commodity $i$ in the game $\mathcal{E}^{1}$ by adopting the above strategy. It can be easily verified that:

$$
\beta_{h}^{i, 1}+\beta_{h}^{i, 2} \geq \hat{\beta}_{h}^{i}
$$

- Step III Now consider the commodities $i \in L_{2}$ (i.e., those commodities for which the consumer is a net seller in both trading posts).

In this case the consumer's net trade is : $-\left(\theta_{h}^{i, 1}+\theta_{h}^{i, 2}\right)$ and the receipts from those sales are:

$\theta_{h}^{i, 1} \cdot \frac{\hat{B}_{-h}^{i, 1}}{\hat{Q}_{-h}^{i, 1}+\theta_{h}^{i, 1}}+\theta_{h}^{i, 2} \cdot \frac{\hat{B}_{-h}^{i, 2}}{\hat{Q}_{-h}^{i, 2}+\theta_{h}^{i, 2}}=\theta_{h}^{i, 1} \cdot \frac{t_{i} \cdot B_{-h}^{i}}{t_{i} \cdot Q_{-h}^{i}+\theta_{h}^{i, 1}}+\theta_{h}^{i, 2} \cdot \frac{\left(1-t_{i}\right) \cdot B_{-h}^{i}}{\left(1-t_{i}\right) \cdot Q_{-h}^{i}+\theta_{h}^{i, 2}}$

In the game $\mathcal{E}^{1}$ the same revenue could be raised via the following offer:

$$
\hat{\theta}_{h}^{i} \cdot \frac{B_{-h}^{i}}{Q_{-h}^{i}+\hat{\theta}_{h}^{i}}=\theta_{h}^{i, 1} \cdot \frac{t_{i} \cdot B_{-h}^{i}}{t_{i} \cdot Q_{-h}^{i}+\theta_{h}^{i, 1}}+\theta_{h}^{i, 2} \cdot \frac{\left(1-t_{i}\right) \cdot B_{-h}^{i}}{\left(1-t_{i}\right) \cdot Q_{-h}^{i}+\theta_{h}^{i, 2}}
$$

This equation can be readily solved to obtain:

$$
\hat{\theta}_{h}^{i}=\frac{t_{i} \cdot\left(1-t_{i}\right) \cdot Q_{-h}^{i} \cdot\left(\theta_{h}^{i, 1}+\theta_{h}^{i, 2}\right)+\theta_{h}^{i, 1} \cdot \theta_{h}^{i, 2}}{t_{i} \cdot\left(1-t_{i}\right) \cdot Q_{-h}^{i}+\left(1-t_{i}\right)^{2} \cdot \theta_{h}^{i, 1}+t_{i}^{2} \cdot \theta_{h}^{i, 2}}
$$

It can be verified that:

$$
\theta_{h}^{i, 1}+\theta_{h}^{i, 2} \geq \hat{\theta}_{h}^{i}
$$

Therefore, this strategy would give the consumer a net trade in commodities $i \in L_{2}$ that would be at least as big as the one with offers in two trading posts. 
-Step IV Consider now the strategy $\left(\hat{\beta}_{h}, \hat{\theta}_{h}\right) \in \Re_{+}^{L}$ which is defined as follows:

$$
\left(\hat{\beta}_{h}, \hat{\theta}_{h}\right)=\left\{\begin{array}{l}
\left(\frac{t_{i \cdot} \cdot\left(1-t_{i}\right) \cdot B_{-h}^{i} \cdot\left(\beta_{h}^{i, 1}+\beta_{h}^{i, 2}\right)+\beta_{h}^{i, 1} \cdot \beta_{h}^{i, 2}}{t_{i} \cdot\left(1-t_{i}\right) \cdot B_{-h}^{i}+\left(1-t_{i}\right)^{2} \cdot \beta_{h}^{i, 1}+t_{i}^{2} \cdot \beta_{h}^{i, 2}}, 0\right) i \in L_{1} \\
\left(0, \frac{t_{i} \cdot\left(1-t_{i}\right) \cdot Q_{-h}^{i} \cdot\left(\theta_{h}^{i, s}+\theta_{h}^{i, r}\right)+\theta_{h}^{i, s} \cdot \theta_{h}^{i, r}}{t_{i} \cdot\left(1-t_{i}\right) \cdot Q_{-h}^{i}+\left(1-t_{i}\right)^{2} \cdot \theta_{h}^{i, s}+t_{i}^{2} \cdot \theta_{h}^{i, r}}\right) i \in L_{2}
\end{array}\right.
$$

Using (7) and (8) from above, it turns out that this strategy is budget feasible in the game $\mathcal{E}^{\mathbf{1}}$ :

$$
\begin{aligned}
\sum_{i=1}^{L}\left(\frac{B_{-h}^{i}+\hat{\beta}_{h}^{i}}{Q_{-h}^{i}+\hat{\theta}_{h}^{i}} \cdot \hat{\theta}_{h}^{i}\right) & =\sum_{i \in L_{1}}\left(\frac{B_{-h}^{i}+\hat{\beta}_{h}^{i}}{Q_{-h}^{i}+\hat{\theta}_{h}^{i}} \cdot \hat{\theta}_{h}^{i}\right)+\sum_{i \in L_{2}}\left(\frac{B_{-h}^{i}+\hat{\beta}_{h}^{i}}{Q_{-h}^{i}+\hat{\theta}_{h}^{i}} \cdot \hat{\theta}_{h}^{i}\right) \\
& =\sum_{i \in L_{2}}\left(\frac{B_{-h}^{i}}{Q_{-h}^{i}+\hat{\theta}_{h}^{i}}\right) \cdot \hat{\theta}_{h}^{i} \\
& =\sum_{i \in L_{2}}\left(\frac{t_{i} \cdot B_{-h}^{i}}{t_{i} \cdot Q_{-h}^{i}+\theta_{h}^{i, 1}} \cdot \theta_{h}^{i, 1}+\frac{\left(1-t_{i}\right) \cdot B_{-h}^{i}}{\left(1-t_{i}\right) \cdot Q_{-h}^{i}+\theta_{h}^{i, 2}} \cdot \theta_{h}^{i, 2}\right) \\
& \geq \sum_{i \in L_{1}}\left(\beta_{h}^{i, 1}+\beta_{h}^{i, 2}\right) \\
& \geq \sum_{i \in L_{1}} \hat{\beta}_{h}^{i} \\
& =\sum_{i=1}^{L} \hat{\beta}_{h}^{i}
\end{aligned}
$$

Recall that for each $i=1,2, \ldots, L$ :

$$
x_{h}^{i}\left(\hat{\beta}_{h}^{i}, \hat{\theta}_{h}^{i}, B_{-h}^{i}, Q_{-h}^{i}\right) \geq x_{h}^{i}\left(\left(\beta_{h}^{i, s}, \theta_{h}^{i, s}, \hat{B}_{-h}^{i, s}, \hat{Q}_{-h}^{i, s}\right)_{s=1}^{2}\right)
$$

By the monotonicity of preferences we have that:

$$
\begin{aligned}
u_{h}\left(\left(x_{h}^{i}\left(\hat{\beta}_{h}^{i}, \hat{\theta}_{h}^{i}, B_{-h}^{i}, Q_{-h}^{i}\right)\right)_{i=1}^{L}\right) & \geq u_{h}\left(\left(x_{h}^{i}\left(\left(\beta_{h}^{i, s}, \theta_{h}^{i, s}, \hat{B}_{-h}^{i, s}, \hat{Q}_{-h}^{i, s}\right)_{s=1}^{2}\right)\right)_{i=1}^{L}\right) \\
& >u_{h}\left(\left(x_{h}^{i}\left(\left(\hat{b}_{h}^{i, s}, \hat{q}_{h}^{i, s}, \hat{B}_{-h}^{i, s}, \hat{Q}_{-h}^{i, s}\right)_{s=1}^{2}\right)\right)_{i=1}^{L}\right) \\
& =u_{h}\left(\left(x_{h}^{i}\left(b_{h}^{i}, q_{h}^{i}, B_{-h}^{i}, Q_{-h}^{i}\right)\right)_{i=1}^{L}\right)
\end{aligned}
$$

which contradicts the fact that $\left(b_{h}, q_{h}\right)_{h \in H}$ is a Nash equilibrium for the game $\mathcal{E}^{\mathbf{1}}$. So our original hypothesis is ruled out and our claim is proved 
Proof of proposition 6:

We prove that $x \in \mathbf{E}\left(\mathcal{E}^{\mathbf{1}}\right)$. The rest follows as a consequence of the previous result.

Let $(b, q) \in \mathbf{N E}\left(\mathcal{E}^{\mathbf{t}}\right)$ be the profile of strategies which gives rise to the allocation $x$. By assumption: $p^{i, s}=p^{i, r}=p^{i}$ for $s \neq r$. Recall that by corollary ?? this implies that for each $h \in H$ :

$$
\frac{B_{-h}^{i, 1}}{Q_{-h}^{i, 1}}=\frac{B_{-h}^{i, 2}}{Q_{-h}^{i, 2}}=\ldots=\frac{B_{-h}^{i, T_{i}}}{Q_{-h}^{i, T_{i}}}
$$

For each $h \in H$ consider the strategy $\left(\hat{b}_{h}, \hat{q}_{h}\right) \in \Re_{+}^{L} \times \Re_{+}^{L}$ defined as follows:

$$
\hat{b}_{h}^{i}=\sum_{s=1}^{T_{i}} b_{h}^{i, s}, \hat{q}_{h}^{i}=\sum_{s=1}^{T_{i}} q_{h}^{i, s}
$$

Certainly, $\left(\hat{b}_{h}, \hat{q}_{h}\right) \in S^{\mathbf{1}}$ for all $h \in H$. Furthermore, it is easy to see that $\left(\hat{b}_{h}, \hat{q}_{h}\right)$ is budget feasible for each $h \in H$. With this profile agent $h$ obtains the net trade:

$$
\begin{aligned}
z_{h}^{i} & =\hat{b}_{h}^{i} \cdot \frac{\sum_{h \in H} \hat{q}_{h}^{i}}{\sum_{h \in H} \hat{b}_{h}^{i}}-\hat{q}_{h}^{i} \\
& =\left(\sum_{s=1}^{T_{i}} b_{h}^{i, s}\right) \cdot \frac{\sum_{h \in H} \sum_{s=1}^{T_{i}} q_{h}^{i, s}}{\sum_{h \in H} \sum_{s=1}^{T_{i}} b_{h}^{i, s}}-\sum_{s=1}^{T_{i}} q_{h}^{i, s} \\
& =\left(\sum_{s=1}^{T_{i}} b_{h}^{i, s}\right) \cdot \frac{\sum_{s=1}^{T_{i}} Q^{i, s}}{\sum_{s=1}^{T_{i}} B^{i, s}}-\sum_{s=1}^{T_{i}} q_{h}^{i, s} \\
& =\frac{\sum_{s=1}^{T_{i}} b_{h}^{i, s}}{p^{i}}-\sum_{s=1}^{T_{i}} q_{h}^{i, s} \\
& =\sum_{s=1}^{T_{i}} z_{h}^{i, s}
\end{aligned}
$$

Thus, with the profile $\left(\hat{b}_{h}, \hat{q}_{h}\right)_{h \in H}$ in the game $\mathcal{E}^{\mathbf{1}}$ each individual obtains the same consumption allocation as in the game $\mathcal{E}^{\mathbf{t}}$. It follows that:

$$
u_{h}\left(\left(x_{h}^{i}\left(\hat{b}_{h}^{i}, \hat{q}_{h}^{i}, \hat{B}_{-h}^{i}, \hat{Q}_{-h}^{i}\right)\right)_{i=1}^{L}\right)=u_{h}\left(\left(x_{h}^{i}\left(\left(b_{h}^{i, s}, q_{h}^{i, s}, B_{-h}^{i, s}, Q_{-h}^{i, s}\right)_{s=1}^{T_{i}}\right)\right)_{i=1}^{L}\right)(12
$$


We claim that $(\hat{b}, \hat{q}) \in \mathbf{N E}\left(\mathcal{E}^{\mathbf{1}}\right)$.

Suppose not. Then there would exist $h \in H$ and a budget feasible $\left(\beta_{h}, \theta_{h}\right) \in S_{h}^{\mathbf{1}}$ such that:

$$
u_{h}\left(\left(x_{h}^{i}\left(\beta_{h}^{i}, \theta_{h}^{i}, \hat{B}_{-h}^{i}, \hat{Q}_{-h}^{i}\right)\right)_{i=1}^{L}\right)>u_{h}\left(\left(x_{h}^{i}\left(\hat{b}_{h}^{i}, \hat{q}_{h}^{i}, \hat{B}_{-h}^{i}, \hat{Q}_{-h}^{i}\right)\right)_{i=1}^{L}\right)
$$

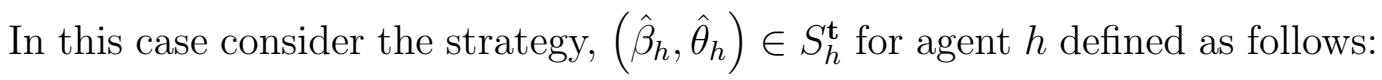
for each $i=1,2, \ldots, L$ and $s=1,2, \ldots, T_{i}$

$$
\hat{\beta}_{h}^{i, s}=\frac{B_{-h}^{i, s}}{\sum_{i=1}^{T_{i}} B_{-h}^{i, s}} \cdot \beta_{h}^{i}, \quad \hat{\theta}_{h}^{i, s}=\frac{Q_{-h}^{i, s}}{\sum_{i=1}^{T_{i}} Q_{-h}^{i, s}} \cdot \theta_{h}^{i}
$$

By (11) and the fact that $\left(\beta_{h}, \theta_{h}\right)$ has been assumed feasible, it follows that given $\left(b_{a}, q_{a}\right)_{a \neq h}$, the strategy defined in (14) is budget feasible for the individual $h$. Furthermore, with that strategy in the game $\mathcal{E}^{\mathbf{t}}$ this agent would obtain the net trade:

$$
\hat{z}_{h}^{i}=\sum_{i=1}^{T_{i}} \hat{\beta}_{h}^{i, s} \frac{Q_{-h}^{i, s}+\hat{\theta}_{h}^{i, s}}{B_{-h}^{i, s}+\hat{\beta}_{h}^{i, s}}-\sum_{i=1}^{T_{i}} \hat{\theta}_{h}^{i, s}
$$

Using (10) we have: $\frac{Q_{-h}^{i, s}+\hat{\theta}_{h}^{i, s}}{B_{-h}^{i, s}+\hat{\beta}_{h}^{\hat{i}, s}}=\frac{Q_{-h}^{i, r}+\hat{\theta}_{h}^{i, r}}{B_{-h}^{i, r}+\hat{\beta}_{h}^{i, r}}$ for all $s \neq r$. It follows that:

$$
\forall s=1,2, \ldots, T_{i}, \quad \frac{Q_{-h}^{i, s}+\hat{\theta}_{h}^{i, s}}{B_{-h}^{i, s}+\hat{\beta}_{h}^{i, s}}=\frac{\sum_{i=1}^{T_{i}} Q_{-h}^{i, s}+\hat{\theta}_{h}^{i, s}}{\sum_{i=1}^{T_{i}} B_{-h}^{i, s}+\hat{\beta}_{h}^{i, s}}=\frac{Q_{-h}^{i}+\theta_{h}^{i}}{B_{-h}^{i}+\beta_{h}^{i}}
$$

Substituting this into (15) above we conclude that:

$$
\hat{z}_{h}^{i}=\frac{Q_{-h}^{i}+\theta_{h}^{i}}{B_{-h}^{i}+\beta_{h}^{i}} \cdot\left(\sum_{i=1}^{T_{i}} \hat{\beta}_{h}^{i, s}\right)-\sum_{i=1}^{T_{i}} \hat{\theta}_{h}^{i, s}=\beta_{h}^{i} \cdot \frac{Q_{-h}^{i}+\theta_{h}^{i}}{B_{-h}^{i}+\beta_{h}^{i}}-\theta_{h}^{i}
$$

But the last term is exactly the net trade that $h$ receives with the strategy $\left(\beta_{h}, \theta_{h}\right)$ in the game $\mathcal{E}^{\mathbf{1}}$. Hence, we have:

$$
u_{h}\left(\left(x_{h}^{i}\left(\left(\hat{\beta}_{h}^{i, s}, \hat{\theta}_{h}^{i, s}, B_{-h}^{i, s}, Q_{-h}^{i, s}\right)_{s=1}^{T_{i}}\right)\right)_{i=1}^{L}\right)=u_{h}\left(\left(x_{h}^{i}\left(\beta_{h}^{i}, \theta_{h}^{i}, \hat{B}_{-h}^{i}, \hat{Q}_{-h}^{i}\right)\right)_{i=1}^{L}\right)
$$


Combining the last equation with (12) and (13) from above we conclude that:

$u_{h}\left(\left(x_{h}^{i}\left(\left(\hat{\beta}_{h}^{i, s}, \hat{\theta}_{h}^{i, s}, B_{-h}^{i, s}, Q_{-h}^{i, s}\right)_{s=1}^{T_{i}}\right)\right)_{i=1}^{L}\right)>u_{h}\left(\left(x_{h}^{i}\left(\left(b_{h}^{i, s}, q_{h}^{i, s}, B_{-h}^{i, s}, Q_{-h}^{i, s}\right)_{s=1}^{T_{i}}\right)\right)_{i=1}^{L}\right)$

which contradicts the hypothesis that $(b, q) \in \mathbf{N E}\left(\mathcal{E}^{\mathbf{t}}\right)$ 


\section{References}

[1] Amir, R., S. Sahi, M. Shubik and S. Yao (1990): "A Strategic Market Game with Complete Markets," Journal of Economic Theory, 51, 126-143.

[2] Bloch F. and H.Ferrer (1999): "Trade Fragmentation and Coordination in Bilateral Oligopolies," C.O.R.E discusion paper No 9908.

[3] Dubey, P. and M. Shubik (1978): "A Theory of Money and Financial Institutions. The Non-cooperative Equilibria of a Closed Economy with Market Supply and Bidding Strategies," Journal of Economic Theory, 17, 1- 20.

[4] Koutsougeras L.C. (1999): "A Remark on the Number of Trading Spots in Strategic Market Games" C.O.R.E discussion paper No 9904.

[5] Koutsougeras L.C. (2001): "Non Walrasian Equilibria and the Law of One Price" Journal of Economic Theory, forthcoming.

[6] Mas-Colell, A. (1982): The Cournotian Foundations of Walrasian Equilibrium Theory: An Exposition of Recent Theory, in Advances in Economic Theory, ed. W. Hildenbrand, Cambridge University Press, 183-224.

[7] Peck, J., K. Shell and S. Spear (1992): "The Market Game: Existence and Structure of Equilibrium," Journal of Mathematical Economics, 21, 271-299.

[8] Postlewaite, A. and D. Schmeidler (1978): "Approximate efficiency of Nonwalrasian Nash Equilibria," Econometrica, 46, 127-135.

[9] Shapley, L.S. and M. Shubik (1975): "Trade Using One Commodity as a Means of Payment," Journal of Political Economy, 85, 937-968. 